\title{
Rotational Coherence Encoded in an "Air-Laser" Spectrum of Nitrogen Molecular Ions in an Intense Laser Field
}

\author{
Haisu Zhang, ${ }^{1,3}$ Chenrui Jing,,${ }^{1,3}$ Jinping Yao, ${ }^{1}$ Guihua Li, ${ }^{1}$ Bin Zeng, ${ }^{1}$ Wei Chu,${ }^{1}$ Jielei Ni, ${ }^{1,3}$ Hongqiang Xie, ${ }^{1,3}$ \\ Huailiang $\mathrm{Xu},{ }^{2, *}$ See Leang Chin, ${ }^{4}$ Kaoru Yamanouchi, ${ }^{5}$ Ya Cheng, ${ }^{1, \dagger}$ and Zhizhan $\mathrm{Xu}^{1, \$}$ \\ ${ }^{1}$ State Key Laboratory of High Field Laser Physics, Shanghai Institute of Optics and Fine Mechanics, Chinese Academy of Sciences, \\ P.O. Box 800-211, Shanghai 201800, China \\ ${ }^{2}$ State Key Laboratory on Integrated Optoelectronics, College of Electronic Science and Engineering, Jilin University, \\ Changchun 130012, China \\ ${ }^{3}$ University of Chinese Academy of Sciences, Beijing 100049, China \\ ${ }^{4}$ Center for Optics, Photonics and Laser, Université Laval, Quebec City, Quebec G1V 0A6, Canada \\ ${ }^{5}$ Department of Chemistry, School of Science, The University of Tokyo, 7-3-1 Hongo, Bunkyo-ku, Tokyo, 113-0033, Japan
}

(Received 17 May 2013; revised manuscript received 3 June 2013; published 29 October 2013)

\begin{abstract}
We investigate lasing action in aligned nitrogen molecular ions $\left(\mathrm{N}_{2}{ }^{+}\right)$produced in an intense laser field. We find that, besides the population inversion between the $\mathrm{B}^{2} \Sigma_{u}{ }^{+}-\mathrm{X}^{2} \Sigma_{g}{ }^{+}$states, which is responsible for the observed simulated amplification of a seed pulse, a rotational wave packet in the ground vibrational state $(v=0)$ of the excited electronic $\mathrm{B}^{2} \Sigma_{u}{ }^{+}$state has been created in $\mathrm{N}_{2}{ }^{+}$. The rotational coherence can faithfully encode its characteristics into the amplified seed pulses, enabling reconstruction of rotational wave packets of molecules in a single-shot detection manner from the frequency-resolved laser spectrum. Our results suggest that the air laser can potentially provide a promising tool for remote characterization of coherent molecular rotational wave packets.
\end{abstract}

DOI: 10.1103/PhysRevX.3.041009

\section{INTRODUCTION}

In the presence of strong laser fields, molecules can exhibit many intriguing behaviors, such as high-order harmonic generation [1], above-threshold ionization and dissociation [2], bond softening and hardening [3], and rotational excitation and molecular alignment [4]. Strong-field molecular physics has become an important subject of contemporary physics and has already triggered a broad range of applications, including molecular orbital imaging [5,6], coherent x-ray sources [7], attosecond chemistry [8], filament control [9], and so forth. Recently, it was discovered that by exposure to ultrashort intense laser pulses, first molecules can be photoionized, and then a population inversion can be instantaneously (i.e., within the duration of the excitation pulses, which is typically a few tens of femtoseconds) established between the excited and ground electronic states of the molecular ions [10-13]. This mechanism leads to either amplified-spontaneousemission (ASE) air lasing in the backward direction if there is no seeding pulses participating in the process [14], or coherent narrow-bandwidth emission in the forward direction in the presence of self-generated or exter-

\footnotetext{
*huailiang@jlu.edu.cn

ya.cheng@siom.ac.cn

${ }^{\ddagger}$ zzxu@mail.shcnc.ac.cn
}

Published by the American Physical Society under the terms of the Creative Commons Attribution 3.0 License. Further distribution of this work must maintain attribution to the author(s) and the published article's title, journal citation, and DOI. nally injected seed pulses [10-12]. It is noteworthy that, although air lasing due to ASE has also been demonstrated with picosecond pump lasers [15], the mechanism there is clearly understood as a resonant two-photon dissociation of molecular oxygen, followed by resonant two-photon excitation of atomic oxygen fragments, which is completely different from the mechanism of the air lasing observed in nitrogen molecules with femtosecond pump lasers.

In this paper, we show that, surprisingly, the abovementioned externally seeded air laser [10-12] can be strongly affected by rotational wave packets of molecular ions in the ground vibrational state $(v=0)$ of the excited electronic $\mathrm{B}^{2} \Sigma_{u}{ }^{+}$state of $\mathrm{N}_{2}{ }^{+}$produced in the femtosecond-laser-induced plasma spark. In our experiment, this effect has been observed in both the frequency domain and the time domain. In the frequency domain, we have observed spectral peaks corresponding to the $R$-branch $(\Delta J=1)$ transitions from the discrete rotational levels of the upper $\mathrm{B}^{2} \Sigma_{u}{ }^{+}$state in the laser spectrum. In the time domain, we have observed periodic modulations of the laser intensity by changing the time delay between the pump and probe pulses. Further theoretical calculations show that, based on these measurements, quantitative characterization and, consequently, direct reconstruction of a rotational wave packet of molecules can be achieved in a single-shot detection manner. Previously, signatures of rotational coherence of molecules observed in a plasma channel produced by femtosecond laser filamentation have been found, mainly by effects induced either by a macroscopic transient-refractive-index change of the media or by 
energy transfer from the light field to the molecules (i.e., redshift of the driving spectrum) during the propagation [16]. The evidence obtained in such "indirect" observations contains entangled contributions from different unidentified rotational states and does not allow for straightforward decoding of the rotational states distribution for wave-packet reconstruction.

\section{EXPERIMENTS}

\section{A. Experimental schematic}

The frequency-resolved seed-laser-amplification spectra and their signal modulations in the time domain were measured using a pump-probe scheme similar to the one described in Ref. [11]. In brief, a linearly polarized laser pulse $(800 \mathrm{~nm}, 40 \mathrm{fs}, 1 \mathrm{kHz})$ from a Ti:sapphire laser system (Legend Elite-Duo, Coherent, Inc.) was split into two by a 50:50 beam splitter; one part was used as the pump to produce a plasma channel of $\mathrm{N}_{2}$, and the other was frequency doubled with a $100-\mu \mathrm{m}$-thickness $\beta$-barium borate crystal. The generated $400-\mathrm{nm}$ pulses were then used as a probe to seed the population-inverted $\mathrm{N}_{2}{ }^{+}$in the plasma. A dichroic mirror with high reflectivity at $400 \mathrm{~nm}$ and high transmission at $800 \mathrm{~nm}$ was used to remove the $800-\mathrm{nm}$ fundamental from the probe. The probe pulse first passed through a polarizer for generating a linearly polarized beam. A half-wave plate was used to control the polarization direction of the pump pulse to be parallel or perpendicular to that of the probe pulse. A variable delay line with a temporal resolution of $16.67 \mathrm{fs}$ was used to adjust the delay between the pump and probe pulses. The pump and probe beams were recombined collinearly with a second dichroic mirror with high reflectivity at $400 \mathrm{~nm}$ and high transmission at $800 \mathrm{~nm}$. They were then focused by an $f=40$-cm fused-silica lens into a gas chamber filled with pure $\mathrm{N}_{2}$ at 20 mbar. The energies of the probe and pump pulses just before the chamber were measured to be $0.1 \mu \mathrm{J}$ and $2.1 \mathrm{~mJ}$, respectively. After passing through the chamber, the pump and probe pulses were collimated by an $f=30$-cm lens and then separated by a third dichroic mirror. The probe-pulse signal was collected by an integration sphere and sent by an optical fiber into a 1200-grooves/mm grating spectrometer (Shamrock 303i, Andor) equipped with a CCD camera.

\section{B. Rotational information in an "air-laser" spectrum}

Figure 1 illustrates the spectra of the probe pulse obtained after passing through the gas chamber with (blue and red lines) and without (green line) the pump pulse. We see that a very strong peak with a central wavelength at about $391 \mathrm{~nm}$ and a very narrow full-width-half-maximum (FWHM) linewidth of $0.3 \mathrm{~nm}$ appears in the presence of the pump pulse when compared with the spectrum obtained without the pump pulse. The strong 391-nm emission corresponding to the rotational $P$ branch bandhead $(\Delta J=-1)$ in the electronic transition $\mathrm{B}^{2} \Sigma_{\mathrm{u}}{ }^{+} \rightarrow$ $\mathrm{X}^{2} \Sigma_{\mathrm{g}}{ }^{+}(0 \rightarrow 0)$ of $\mathrm{N}_{2}{ }^{+}$[17] is ascribed to amplification of the spectral portion of the probe pulse due to the population inversion of $\mathrm{N}_{2}{ }^{+}$established in the plasma channel by the pump-laser excitation [10-13].

In addition, a previously unobserved amplification in the blue side of the 391-nm line can be clearly seen in Fig. 1. By a careful examination of this region, spectral peaks corresponding to the $R$-branch $(\Delta J=1)$ transitions are frequency resolved, as shown in the inset of Fig. 1, in which the integer numbers indicate the discrete rotational levels of the upper $\mathrm{B}^{2} \Sigma_{\mathrm{u}}{ }^{+}$state. The fact that only the rotational states of odd numbers of $J$ levels were observed is probably a result of the relative abundance of the orthoand para- $\mathrm{N}_{2}$ [18]. As shown in the inset of Fig. 1, the rotational states of the $R$ branch with numbers of $J$ up to 29 have been observed, with the strongest line at $J_{\max }=15$. The shift of the rotational-state distribution toward the higher $J$ levels as compared with that of $J_{\max }=7$ at room temperature by the Boltzmann distribution comes from the coherent nonresonant Raman rotational excitation of the ionized $\mathrm{N}_{2}{ }^{+}$molecules in the

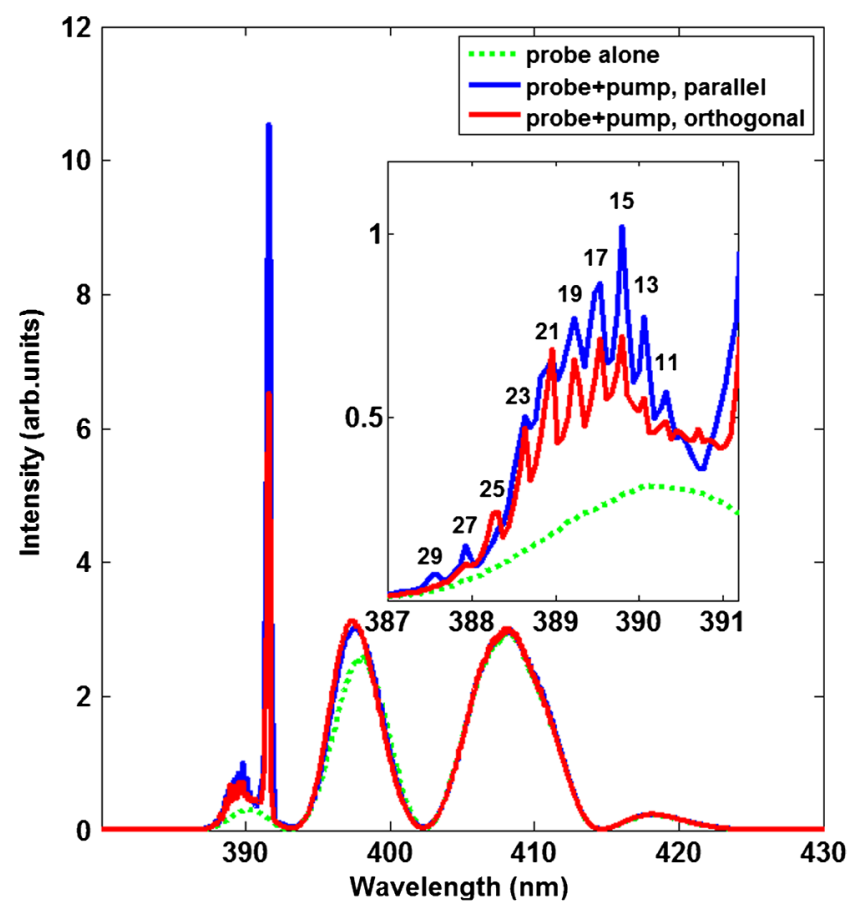

FIG. 1. The forward-probe-pulse spectra captured with the polarization direction of the probe pulse parallel (blue line) or perpendicular (red line) to the pump pulse. The inset shows the enlarged spectral region in the spectral range of 387-391 nm corresponding to the $R$-branch transition, and the numbers label the upper rotational levels. The estimated intensities of the pump and the probe pulses are on the orders of $10^{15} \mathrm{~W} / \mathrm{cm}^{2}$ and $10^{12} \mathrm{~W} / \mathrm{cm}^{2}$, respectively, by assuming a linear propagation condition. 
pump-laser pulse [19]. It should be mentioned that, although the spectral modulations in Fig. 1 that define the $R$ branch require a longer time to resolve themselves than the duration of the probe pulse, in consideration of the time-bandwidth product, previously we have measured the emission time of the laser to be several picoseconds, which resolves this issue [11].

We notice in Fig. 1 that in the presence of the pump pulse, the amplified signal is stronger when the probe pulse has a polarization direction parallel to that of the pump pulse. This is because when the ensemble of $\mathrm{N}_{2}{ }^{+}$is excited into states with higher rotational angular momenta, the projection of the angular momentum of each rotational state onto the polarization direction of the pump pulse (the azimuthal quantum number $M$ ) is preserved as the values of initial lower rotational states, owing to the cylindrically symmetry with respect to the polarization direction of the pump pulse in the Raman rotational excitation process [20]. The $M$ values after the rotational excitation by the pump pulse are limited by the initial rotational quantum numbers with $|M| \leq J_{i}$, where $J_{i}$ is the initial rotational quantum number. Since $J_{i}$ is much lower than the final rotational quantum number $J_{f}$, it leads to an anisotropic distribution of the molecules populated mostly on $\left|J_{f},\right| M\left|\ll J_{f}\right\rangle$ sublevels for a specific rotational state $J_{f}$. For linear and symmetric top molecules, a $P$-branch $(J \rightarrow J+1)$ or an $R$-branch $(J \rightarrow J-1)$ transition is more likely to occur with a smaller $M$ value [21]. Therefore, when the probe pulse has a polarization direction parallel or perpendicular to that of the pump pulse, it will see a small or large $M$ value, giving rise to the discrepancy of the $P$-branch or $R$-branch transition intensities in the two polarization cases.

\section{Periodic modulation of laser signals in the pump-probe measurements}

The rotational-state distribution of $\mathrm{B}^{2} \Sigma_{\mathrm{u}}^{+}$shown in Fig. 1 is expected to be coherently populated, and it forms a coherent rotational wave packet because of the alignment of molecules. Generally speaking, alignment of $\mathrm{N}_{2}{ }^{+}$can be achieved either by an impulsive Raman excitation [18] or by a preferential ionization of $\mathrm{N}_{2}$ molecules whose axes are parallel to the laser polarization [22], or by both of them. With the alignment, the rotational coherence of the wave packet would survive after the turnoff of the pump pulse, and the ensuing free evolution of the rotational wave packet causes the constituent rotational eigenstates to dephase and rephase periodically, giving rise to the wellknown revival of molecular alignment [23].

To verify the rotational coherence, we measured the signal intensity of the $P$-branch bandhead at around $391 \mathrm{~nm}$ as a function of the time delay between the pump and probe pulses, as shown in Fig. 2(a), for the polarization directions of the two pulses to be either parallel (blue line) or perpendicular (red line) to each other, respectively. The zero time delay is set at the instant when the signal intensity is the strongest (i.e., the maximum amplification occurs), and the positive time delay means the probe pulse is behind the pump pulse. We see in Fig. 2(a) that both curves show a rapid increase followed by slow decays, representing the fast growth in the beginning and the gradual damping of the population inversion between the $\mathrm{B}^{2} \Sigma_{\mathrm{u}}{ }^{+}$and $\mathrm{X}^{2} \Sigma_{\mathrm{g}}{ }^{+}(0-0)$ states. By fitting both curves with an exponential decay function $a \times \exp (-t / \Gamma)+b$, the decay times of $\Gamma=3 \pm 0.2 \mathrm{ps}$ and $\Gamma=4 \pm 0.3 \mathrm{ps}$ are extracted, respectively, for the parallel (purple line) and perpendicular (green line) cases, showing good agreement with each other for the decay of the population inversion between the $\mathrm{B}^{2} \Sigma_{\mathrm{u}}{ }^{+}$and $\mathrm{X}^{2} \Sigma_{\mathrm{g}}{ }^{+}$ $(0-0)$ states. Notably, periodic modulations of the signal intensities can be clearly observed in Fig. 2(a), which shows the synchronized antiphase oscillations for the two polarization directions of the pump pulses. The periodic modulations of signals at approximately $2.0 \mathrm{ps}, 4.0 \mathrm{ps}$, $6.0 \mathrm{ps}, 8.0 \mathrm{ps}$, and $10.0 \mathrm{ps}$ can be reasonably assigned to $T_{\text {rot }} / 4, T_{\text {rot }} / 2,3 T_{\text {rot }} / 4, T_{\text {rot }}$, and $5 T_{\text {rot }} / 4$ revivals of the rotational wave packet of the $\mathrm{B}^{2} \Sigma_{\mathrm{u}}{ }^{+}$state of $\mathrm{N}_{2}{ }^{+}$, with $T_{\text {rot }}=(2 B c)^{-1}=8.0 \mathrm{ps}$ being the fundamental rotational period, where $B=2.083 \mathrm{~cm}^{-1}$ is the rotational constant of the electronic $\mathrm{B}^{2} \Sigma_{\mathrm{u}}^{+}$state and $c$ is the velocity of light.
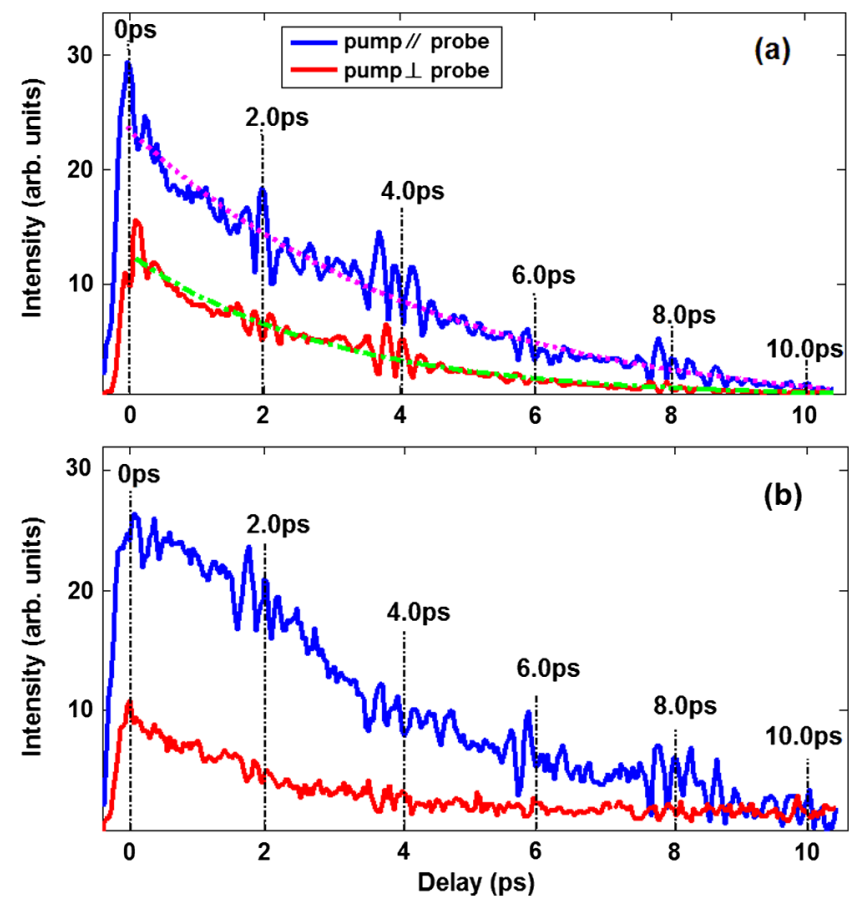

FIG. 2. The signal intensities of (a) the $P$-branch bandhead at around $391 \mathrm{~nm}$ and (b) the $R$-branch band recorded over the range of 387-390.7 $\mathrm{nm}$ as a function of the time delay between the pump and probe pulses for the parallel or perpendicular cases of the polarization directions of the two pulses. The dotted lines are the exponential fits of the experimental curves. The black dotted vertical lines indicate the revival times of $\mathrm{N}_{2}{ }^{+}$. 

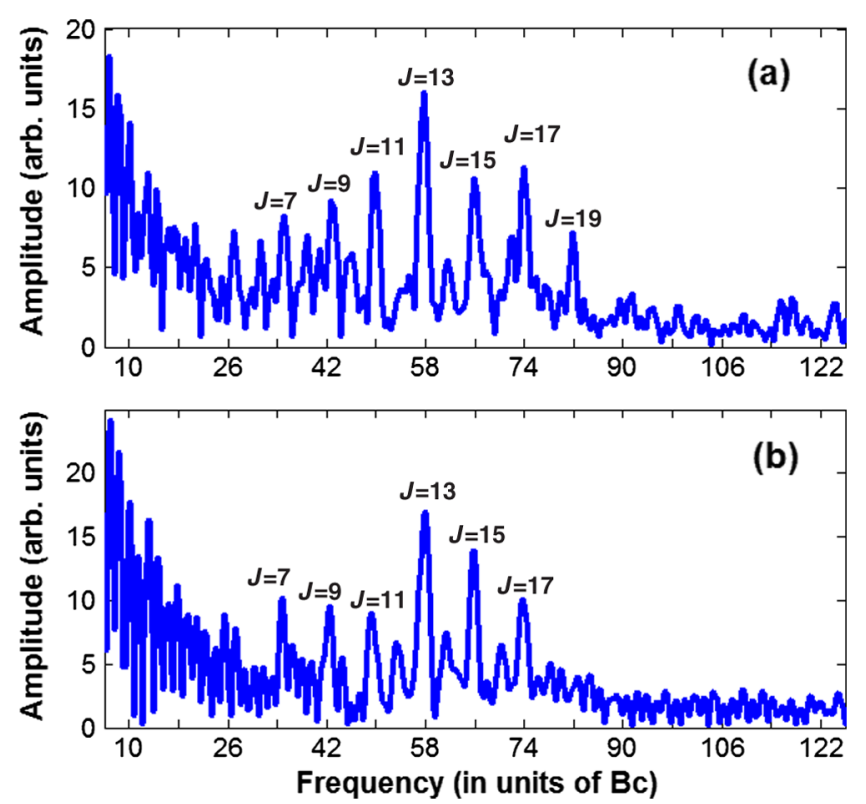

FIG. 3. The Fourier-transform spectra of the oscillation curves in Fig. 2(a) for the parallel (a) and perpendicular (b) cases. The numbers indicate the beat frequency.

To better understand the correlation between the measurements carried out in the time domain [Fig. 2(a)] and frequency domain (inset of Fig. 1), we perform a Fourier transform for the two curves in Fig. 2(a) after removing the exponential decay baselines. The corresponding Fourier spectra for both polarization cases are depicted in Figs. 3(a) and 3(b). We clearly see that these two spectra show remarkably similar frequency distributions, with seven peaks corresponding to the beat frequencies between odd $J$ states (the lower states in the beat terms are labeled). The frequency peak with the largest amplitude belongs to the beat frequency between $J=13$ and $J=15$ rotational states, which is in good agreement with the measured rotational-state distribution in the $R$-branch spectrum (see inset of Fig. 1). We note that several very weak even- $J$ peaks of $\mathrm{N}_{2}{ }^{+}$ions can be seen in Fig. 3, whereas they cannot be observed in Fig. 1. This difference can be attributed to the higher resolution in the time-resolved measurement than that in the frequency-resolved measurement.

\section{THEORETICAL ANALYSIS}

It is well known that the absorption of polarized light by molecules depends on the alignment of the molecule with respect to the polarization direction [24]. Recall that the $\mathrm{B}^{2} \Sigma_{\mathrm{u}}{ }^{+} \rightarrow \mathrm{X}^{2} \Sigma_{\mathrm{g}}{ }^{+}$electronic transition is a parallel transition; the strength of the stimulated emission from molecular $\mathrm{N}_{2}{ }^{+}$aligned parallel to the polarization direction of the probe pulse is stronger than that from perpendicularly aligned $\mathrm{N}_{2}{ }^{+}$. Consequently, the probe pulses injected into the plasma spark with different delay times would see different alignment angles of $\mathrm{N}_{2}{ }^{+}$and thus acquire different amplification efficiencies, resulting in the intensity variations as a function of the alignment of $\mathrm{N}_{2}{ }^{+}$ relative to the polarization direction of the probe pulse. Furthermore, because of the orthogonal polarization directions of the pump pulses in the two polarization cases, the laser-induced alignments of the molecular axis in the two cases are perpendicular to each other for the same delay time, which naturally causes the antiphase responses of the probe pulses. Besides, it should be emphasized that since the $R$-branch and $P$-branch transitions originate from the same upper $J_{f}$ states, the intensity modulations of the $R$ branch and $P$ branch should have the same periodic oscillation patterns. This result is indeed what we have observed, as shown in Fig. 2(b), where the signal intensity of the lines of the $R$-branch band integrated over the spectral range of $387-390.7 \mathrm{~nm}$ is plotted as a function of the delay time between the pump and probe pulses. We find that both curves of the $R$ branches show the modulations with the same oscillation periods and phases as those of the $P$ branches shown in Fig. 2(a).

Based on the above physical picture, we show that the pump-probe experimental results can be fairly well reproduced with a simplified theoretical model as follows. Assume that the rotational wave packet of ionized $\mathrm{N}_{2}{ }^{+}$ after the pump pulse can be written as $\psi_{0}=\sum_{J} a_{J}|J, M\rangle$; the free evolution of the wave packet can then be expressed as $\psi_{0}(t)=\sum_{J} a_{J} e^{-i\left(E_{J} / \hbar\right) t}|J, M\rangle$, with $E_{J}=B J(J+1) h c$ being the energy of the rotational eigenstate (here, we ignore the centrifugal distortions). Then, the spatial information about the orientation of the molecular axes of $\mathrm{N}_{2}{ }^{+}$ at each moment is contained in the superposition state $\psi_{0}(t)$. Furthermore, in this simplified theoretical model, we set the azimuthal quantum number $M=0$. This assumption is mainly based on the following consideration. Before the alignment of the molecules, the $\mathrm{N}_{2}$ molecules are mostly populated at the rotational energy levels with $J$ numbers much lower than those of the rotational energy levels populated by the $\mathrm{N}_{2}{ }^{+}$molecular ions that have been efficiently aligned by the intense pump-laser field. Because of the cylindrical symmetry with respect to the polarization direction of the pump pulse, the initial azimuthal quantum number $M$ of the molecules, which are small before the alignment process, will not be changed by the alignment. Thus, since the rotation of the majority of molecular ions will be highly anisotropic (i.e., mainly confined in the plane perpendicular to the direction of laser propagation) after the laser alignment, we expect that the assumption of $M=0$ should be reasonable. Indeed, as we will see later in Fig. 4, the theoretical calculations based on this assumption agree well with our experimental results; i.e., all the major features in the experimental observations have been captured by our calculations. The time-dependent probability of stimulated emission in the ensemble of the aligned $\mathrm{N}_{2}^{+}$for the parallel transitions between the $\mathrm{B}^{2} \Sigma_{\mathrm{u}}{ }^{+}-\mathrm{X}^{2} \Sigma_{\mathrm{g}}{ }^{+}$states can be written as [24] 

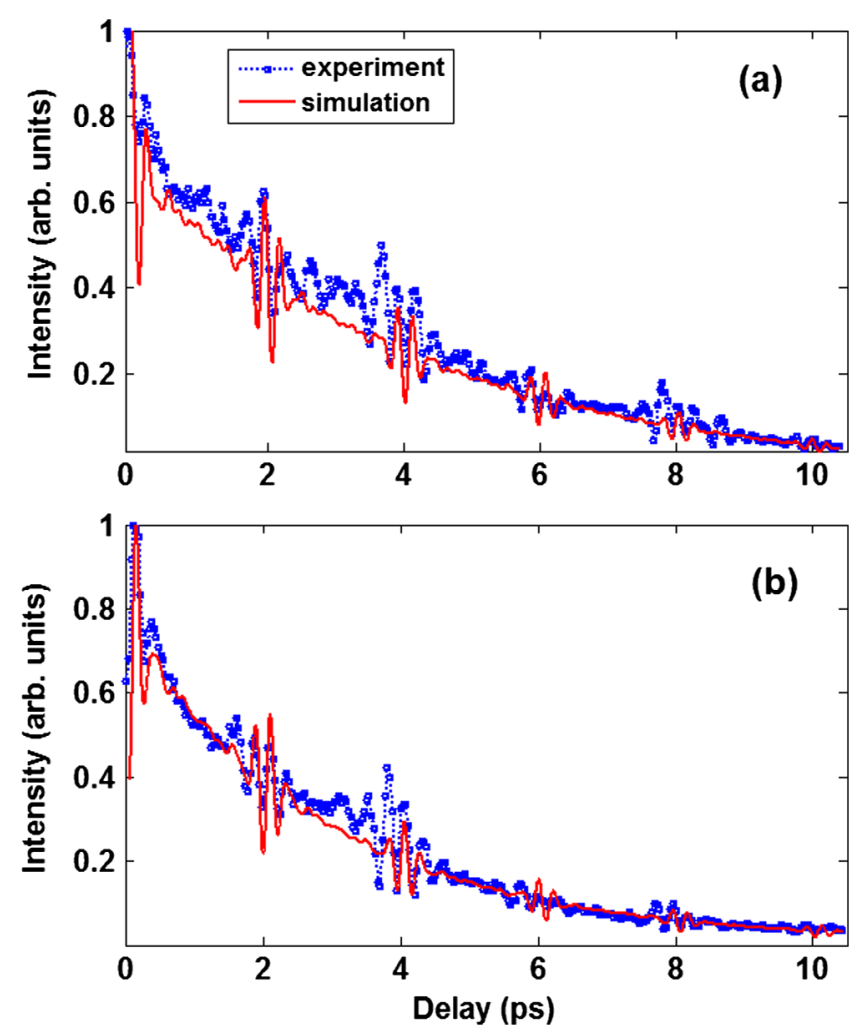

FIG. 4. The experimental (blue dotted lines) and fitted (red solid lines) curves of the signal modulations as a function of the time delay for the parallel (a) and perpendicular (b) cases. Both figures are normalized for clarity.

$$
\begin{aligned}
\left\langle\cos ^{2} \theta\right\rangle(t)= & \sum_{J}\left|a_{J}\right|^{2} C_{J, J, M=0}+\left|a_{J}\right|\left|a_{J+2}\right| \\
& \times \cos \left(\Delta \omega_{J, J+2} t+\phi_{J, J+2}\right) C_{J, J+2, M=0},
\end{aligned}
$$

where $\theta$ is the angle between the molecular axis and the polarization of the probe pulse, $\left|a_{J}\right|$ and $\left|a_{J+2}\right|$ are the respective probability amplitudes of the $|J, M\rangle$ and $|J+2, M\rangle$ states, $\Delta \omega_{J, J+2}=\left(E_{J+2}-E_{J}\right) / \hbar$ is the beat frequency between the $|J, M\rangle$ and $|J+2, M\rangle$ states, $\phi_{J, J+2}$ is the relative phase between the states $|J, M\rangle$ and $|J+2, M\rangle$ at the beginning of free evolution, and $\quad C_{J, J, M=0}=\left\langle J, M\left|\cos ^{2} \theta\right| J, M\right\rangle \quad$ and $C_{J, J+2, M=0}=$ $\left\langle J, M\left|\cos ^{2} \theta\right| J+2, M\right\rangle$ are both constants. Then, we can decide the values of $\left|a_{J}\right|$ by the signal intensities of the $R$-branch band in the seed amplification spectrum of Fig. 1, and the evolution of the rotational wave packet is calculated from the initial largest alignment point after the pump pulse, at which each rotational eigenstate has approximately the same phase as $\phi_{J, J+2}=0$. Therefore, the variation of the signal intensities at different time delays, $\tau$, can be expressed by the function of $\exp (-\tau / \Gamma) \times\left\langle\cos ^{2} \theta\right\rangle(\tau)$. The exponential term $\exp (-\tau / \Gamma)$ describes the decay of population inversion between the $\mathrm{B}^{2} \Sigma_{\mathrm{u}}{ }^{+}$and $\mathrm{X}^{2} \Sigma_{\mathrm{g}}{ }^{+}(0-0)$ states, as mentioned above. Thus, the results of the simu- lations (red solid curves) for the pump and probe pulses in the parallel and orthogonal polarization cases are, respectively, plotted in Figs. 4(a) and 4(b). We see in Figs. 4(a) and 4(b) that all the major features in the experimental curves (blue dotted curves) are qualitatively reproduced with the simulation curves, which confirms the buildup of the rotational wave packet of $\mathrm{N}_{2}{ }^{+}$ions in the plasma spark produced by the intense femtosecond laser pulses. The quantitative discrepancy originates from the fact that either the population inversion or the rotational-state distribution is not perfectly uniform in the plasma spark, and the approximation of $M=0$ (i.e., we have ignored the populations at rotational levels with small $M$ numbers) used in our calculations.

\section{CONCLUSION}

In conclusion, we experimentally show the generation of a rotational wave packet in a population-inverted molecular $\mathrm{N}_{2}{ }^{+}$system in a plasma spark produced by a nearinfrared 800-nm-femtosecond intense laser pulse. The stimulated emissions from such a unique system exhibit periodic modulations in the time domain stemming from the revival of the coherent rotational wave packets in the excited electronic states $\mathrm{B}^{2} \Sigma_{\mathrm{u}}{ }^{+}$. Surprisingly, the rotational-state distribution of the coherent wave packet of the molecular ions has been faithfully mapped onto the frequency-resolved laser spectrum, owing to the relatively long pulse duration (approximately ps) of the laser signal at a wavelength of around $391 \mathrm{~nm}$ [11]. We notice that our observation uniquely covers three time scales, beginning with the tunnel ionization of the molecules on the attosecond time scale, followed by the population-inversion establishment in the molecular ions on the femtosecond time scale, and ending with the amplification of the seed pulses in the aligned molecular ions on the picosecond time scale. Interestingly, all the processes involved are entangled. Our finding provides new evidence toward a complete understanding of the mechanism behind the occurrence of ultrafast lasing in remote air, which is still lacking at the current stage. From the application point of view, the finding will open up the possibility to remotely reconstruct the rotational wave packets produced during the free propagation of an intense femtosecond laser pulse in the atmosphere.

\section{ACKNOWLEDGMENTS}

This work was supported by the National Basic Research Program of China (Grants No. 2011CB808100 and No. 2014CB921300), the National Natural Science Foundation of China (Grants No. 11127901, No. 11134010, No. 60921004, No. 11074098, No. 11204332, No. 60825406, and No. 61235003), New Century Excellent Talent of China (Grant No. NCET-09-0429), and the Basic Research Program of Jilin University. 
[1] R. Vellota, N. Hay, M. B. Mason, M. Castillejo, and J. P. Marangos, High-Order Harmonic Generation in Aligned Molecules, Phys. Rev. Lett. 87, 183901 (2001).

[2] A. Giusti-Suzor, X. He, and O. Atabek, Above-Threshold Dissociation of $\mathrm{H}_{2}{ }^{+}$in Intense Laser Fields, Phys. Rev. Lett. 64, 515 (1990).

[3] P. H. Bucksbaum, A. Zavriyev, H. G. Muller, and D. W. Schumacher, Softening of the $\mathrm{H}_{2}^{+}$Molecular Bond in Intense Laser Fields, Phys. Rev. Lett. 64, 1883 (1990).

[4] H. Sakai, C. P. Safvan, J. J. Larsen, K. M. Hilligsoe, K. Hald, and H. Stapelfeldt, Controlling the Alignment of Neutral Molecules by a Strong Laser Field, J. Chem. Phys. 110, 10235 (1999).

[5] M. Lein, N. Hay, R. Velotta, J. P. Marangos, and P.L. Knight, Interference Effects in High-Order Harmonic Generation with Molecules, Phys. Rev. A 66, 023805 (2002).

[6] T. Kanai, S. Minemoto, and H. Sakai, Quantum Interference during High-Order Harmonic Generation from Aligned Molecules, Nature (London) 435, 470 (2005).

[7] Y. Yu, J. Xu, Y. Fu, H. Xiong, H. Xu, J. P. Yao, B. Zeng, W. Chu, J. Chen, Y. Cheng, and Z.Z. Xu, Single Attosecond Pulse Generation from Aligned Molecules Using TwoColor Polarization Gating, Phys. Rev. A 80, 053423 (2009).

[8] H. J. Worner, J. B. Bertrand, B. Fabre, J. Higuet, H. Ruf, A. Dubrouil, S. Patchkovskii, M. Spanner, Y. Mairesse, V. Blanchet, E. Mével, E. Constant, P. B. Corkum, and D. M. Villeneuve, Conical Intersection Dynamics in $\mathrm{NO}_{2}$ Probed by Homodyne High-Harmonic Spectroscopy, Science 334, 208 (2011).

[9] S. Varma, Y.-H. Chen, and H. M. Milchberg, Trapping and Destruction of Long-Range High-Intensity Optical Filaments by Molecular Quantum Wakes in Air, Phys. Rev. Lett. 101, 205001 (2008).

[10] J.P. Yao, B. Zeng, H. L. Xu, G. H. Li, W. Chu, J. L. Ni, H. S. Zhang, S. L. Chin, Y. Cheng, and Z.Z. Xu, HighBrightness Switchable Multiwavelength Remote Laser in Air, Phys. Rev. A 84, 051802(R) (2011).

[11] J. P. Yao, G. H. Li, C. R. Jing, B. Zeng, W. Chu, J. L. Ni, H. S. Zhang, H. Q. Xie, C. J. Zhang, H. L. Li, H. L. Xu, S. L. Chin, Y. Cheng, and Z.Z. Xu, Remote Creation of Coherent Emissions in Air with Two-Color Ultrafast Laser Pulses, New J. Phys. 15, 023046 (2013).
[12] J. L. Ni, W. Chu, C. R. Jing, H. S. Zhang, B. Zeng, J. P. Yao, G. H. Li, H. Q. Xie, C. J. Zhang, H. L. Xu, S. L. Chin, Y. Cheng, and Z.Z. Xu, Identification of the Physical Mechanism of Generation of Coherent $\mathrm{N}_{2}{ }^{+}$Emissions in Air by Femtosecond Laser Excitation, Opt. Express 21, 8746 (2013).

[13] S.L. Chin, H.L. Xu, Y. Cheng, Z.Z. Xu, and K. Yamanouchi, Natural Population Inversion in a Gaseous Molecular Filament, Chin. Optic. Lett. 11, 013201 (2013).

[14] Q. Luo, W. Liu, S. L. Chin, Lasing Action in Air Induced by Ultra-Fast Laser Filamentation, Appl. Phys. B 76, 337 (2003).

[15] A. Dogariu, J. B. Michael, M. O. Scully, and R. B. Miles, High-Gain Backward Lasing in Air, Science 331, 442 (2011).

[16] J. Wu, H. Cai, H. P. Zeng, and A. Couairon, Femtosecond Filamentation and Pulse Compression in the Wake of Molecular Alignment, Opt. Lett. 33, 2593 (2008).

[17] G. Herzberg, Molecular Spectra and Molecular Structure I: Spectra of Diatomic Molecules (Van Nostrand Company, New York, 1950), 2nd ed.

[18] P. W. Dooley, I. V. Litvinyuk, K. F. Lee, D. M. Rayner, M. Spanner, D. M. Villeneuve, and P. B. Corkum, Direct Imaging of Rotational Wave-Packet Dynamics of Diatomic Molecules, Phys. Rev. A 68, 023406 (2003).

[19] J. Plenge, A. Wirsing, C. Raschpichler, M. Meyer, and E. Ruhl, Chirped Pulse Multiphoton Ionization of Nitrogen: Control of Selective Rotational Excitation in $\mathrm{N}_{2}^{+}\left(\mathrm{B}^{2} \Sigma_{u}^{+}\right)$, J. Chem. Phys. 130, 244313 (2009).

[20] K. Miyazaki, M. Kaku, G. Miyaji, A. Abdurrouf, and F. H. Faisal, Field-Free Alignment of Molecules Observed with High-Order Harmonic Generation, Phys. Rev. Lett. 95, 243903 (2005).

[21] M. J. Weida and C. S. Parmenter, Aligning Symmetric and Asymmetric Top Molecules via Single Photon Excitation, J. Chem. Phys. 107, 7138 (1997).

[22] M. Spanner, S. Patchkovskii, E. Frumker, and P. Corkum, Mechanisms of Two-Color Laser Field-Free Molecular Orientation, Phys. Rev. Lett. 109, 113001 (2012).

[23] H. Stapelfeldt and T. Seideman, Colloquium: Aligning Molecules with Strong Laser Pulses, Rev. Mod. Phys. 75, 543 (2003).

[24] J. J. Larsen, I. W. Larsen, and H. Stapelfeldt, Controlling the Branching Ratio of Photodissociation Using Aligned Molecules, Phys. Rev. Lett. 83, 1123 (1999). 